\title{
A INFLUÊNCIA DO EXCESSO DE PESO NA FORÇA MUSCULAR E NA
}

\author{
FUNCIONALIDADE MULHERES JOVENS
}

\author{
Pâmela Abreu Vargas Barbosa ${ }^{1}$ \\ Amanda Marques Faria ${ }^{2}$ \\ Daniella Alves Vento ${ }^{3}$ \\ Flávio Monteiro Ayres ${ }^{4}$ \\ Cibelle Kayenne Martins Roberto Formiga ${ }^{5}$ \\ Tânia Hamu \\ Recebido em: 10 abr. 2018 \\ Aceito em: 10 abr. 2019
}

RESUMO: O objetivo foi verificar a influência do excesso de peso na força muscular e na funcionalidade de tronco de mulheres jovens. Estudo analítico transversal, com 54 mulheres de 18 a 30 anos, com excesso de peso $(n=25)$, e eutróficas $(n=29)$. A força muscular de tronco foi avaliada pelo dinamômetro isocinético e a funcionalidade de tronco e membros inferiores através do Bunkie Test. A análise dos dados foi realizada por meio do programa SPSS (Sciences Statistical Package for the Social Sciences). Como resultado, o presente estudo identificou que as mulheres com excesso de peso conseguem gerar pico de torque absoluto na $1^{\circ}$ série $(p=0,023)$, na $2^{\circ}$ série $(p=0,009)$, e no impulso na $2^{\circ}$ série $(p=0,009)$ maiores que as mulheres eutróficas. No entanto, quando o pico de torque foi analisado proporcionalmente ao peso corporal, estes valores foram menores, não sendo encontrada diferença entre os grupos $(p>0,05)$. Para o Bunkie Test, o grupo com excesso de peso apresentou pior desempenho na funcionalidade, principalmente nos movimentos de extensão, em decúbito dorsal, dos membros inferiores direito $(p=0,011)$, esquerdo $(p=$ $0,004)$ e na manutenção do tronco em decúbito lateral direito $(p=0,008)$. Conclui-se que $o$ excesso de peso pode alterar diretamente a força muscular e interferir na funcionalidade de tronco de mulheres jovens, sugerindo um desequilíbrio muscular em tronco e membros inferiores.

Palavras-chave: Força Muscular. Obesidade. Funcionalidade.

\section{THE INFLUENCE OF OVERWEIGHT ON MUSCLE STRENGTH AND FUNCTION IN}

\section{YOUNG WOMEN}

ABSTRACT: The aim of this study was to verify the influence of overweight on muscle strength and functionality in young women. This is a cross-sectional analytical study that was conducted with 54 women aged 18 and 30 years - twenty-five (25) were overweight and twenty-nine (29) were eutrophic. Muscle strength was assessed by an isokinetic dynamometer, whereas functionality was assessed by the Bunkie Test. Data analysis was performed using the SPSS program (Sciences Statistical Package for the Social Sciences). As a result, the present study identified that overweight women can

\footnotetext{
1 Universidade Estadual de Goiás.

2 Universidade Estadual de Goiás.

3 Universidade Estadual de Goiás.

${ }^{4}$ Universidade Estadual de Goiás.

${ }^{5}$ Universidade Estadual de Goiás.

6 Universidade Estadual de Goiás.
} 
generate absolute peak torque in the 1 st grade $(p=0.023)$, in the 2 nd grade $(p=$ $0.009)$, and in the 2 nd highest grade $(p=0.009)$ than eutrophic women. However, when the peak torque was analyzed proportionally to the body weight, these values were lower, and no difference was found between the groups ( $p>0.05)$. For the Bunkie Test, the overweight group presented worse performance in the functionality, especially in the extension movements, in the dorsal decubitus position, of the right lower limbs $(p=0.011)$, left $(p=0.004)$ and in the maintenance of the decubitus trunk right side $(p=0.008)$. It is concluded that overweight can directly affect muscle strength and interfere with the trunk function of young women, suggesting a muscle imbalance in the trunk and lower limbs.

Keywords: Muscle Strength. Obesity. Functioning.

\section{INTRODUÇÃO}

Segundo a Organização Mundial de Saúde (WHO, 2017) o excesso de peso, incluindo as condições de sobrepeso e de obesidade, indica o acumulo de gordura anormal ou excessiva considerada prejudicial à saúde. É considerado um problema de saúde pública em todo mundo, sendo uma situação de ampla complexidade, que envolve diversas dimensões sociais e psicológicas, afetando todos os grupos de faixa etária e níveis socioeconômicos.

Em países em desenvolvimento, cerca de 115 milhões de pessoas sofrem de problemas associados à obesidade. $O$ excesso de peso indica um risco grave para doenças não transmissíveis incluindo diabetes mellitus, doenças cardiovasculares, hipertensão e acidentes vasculares encefálicos, e certas formas de câncer (PHILLIPS, 2016). Possui diversos fatores etiológicos, evidenciando os elementos nutricionais, genéticos, psíquico, socioeconômico e o sedentarismo (SANT'ANNA et al., 2015).

Além das condições clínicas e sistêmicas, o excesso de peso está associado a condições musculoesqueléticas como alteração da força muscular, bem como a diminuição da funcionalidade que é a capacidade de desempenhar a atividade básica cotidiana, interferindo assim na qualidade de vida (BATTISTI et al., 2017).

Os efeitos deletérios do excesso de peso na força muscular é uma variante significativa para funcionalidade humana. A análise da força muscular indica a situação real na qual a musculatura do indivíduo se encontra, possibilitando encontrar os desequilíbrios musculares entre músculos agonistas e antagonistas de cada movimento. Nos últimos anos tem se utilizado a avaliação pelo dinamômetro isocinético, considerado padrão ouro para avaliar forçar e o equilíbrio muscular (ZABKA et al., 2011; SILVA NETO, 2010).

A funcionalidade está relacionada com as atividades desempenhadas na vida diária e a participação social de cada indivíduo. É usada como termo de incapacidade, e pode ainda estar associada a limitações de atividade (LOPES; SANTOS, 2015). Neste sentido, a gordura corporal causada pelo acúmulo de tecido adiposo pode influenciar no desequilíbrio biomecânico favorecendo uma sobrecarga e colaborando com o aparecimento de desvios posturais e instabilidade lombar no indivíduo obeso (SIQUEIRA; 
SILVA, 2011).

Estudos demonstram que a obesidade também é capaz de afetar negativamente a força muscular (LOPES et al., 2013; TOSCANO; EGYPTO, 2001). O excesso de peso pode interferir na força muscular de tronco e desencadear sintomas de dor. Diversas alterações biomecânicas são acompanhadas da existência de fraqueza muscular associada ao excesso de peso (SIQUEIRA; SILVA, 2011). Sendo assim, torna-se essencial a caracterização da força muscular e funcionalidade e a sua relação com o acúmulo de peso.

Acredita-se que a força muscular, mensurada pelo dinamômetro isocinético, e a funcionalidade avaliada pelo bunkie test podem sofrer influências do excesso de peso corporal, podendo interferir nas atividades funcionais diárias, na estabilização e na manutenção da postura. Neste contexto, o objetivo do presente estudo foi verificar a influência do excesso de peso na força muscular e na funcionalidade de mulheres jovens.

\section{MATERIAL E MÉTODOS}

Estudo de delineamento transversal, com amostra não probabilística intencional composta por 54 mulheres, acadêmicas da Universidade Estadual de Goiás (UEG), Campus ESEFFEGO, na cidade de Goiânia, Estado de Goiás.

Os seguintes critérios de inclusão foram adotados: mulheres jovens com idade de 18 a 30 anos, deambuladoras comunitárias e independentes, com Índice de Massa Corporal (IMC) variando de eutróficas (IMC entre $18,5 \mathrm{~kg} / \mathrm{m} 2$ a $24,9 \mathrm{~kg} / \mathrm{m} 2$ ) a excesso de peso (IMC acima de $25 \mathrm{~kg} / \mathrm{m} 2)$.

Como critérios de exclusão foram adotados os seguintes: mulheres gestantes, que utilizavam dispositivos auxiliares à marcha, doença clínica ou obstétrica, desordens pulmonares, cardíacas e cognitivas, história de fraturas na coluna ou em membros inferiores, portadoras de doenças neurológicas, do sistema vestibular e usuárias de medicamentos que afetavam o equilíbrio.

A pesquisa foi realizada no Laboratório de Pesquisa em Musculoesquelética (LAPEME) localizado no Campus ESEFFEGO da UEG. O estudo foi realizado em conformidade com as Diretrizes e Normas Regulamentadoras de Pesquisas envolvendo seres humanos (Resolução 466/2012, do Conselho Nacional de Saúde) e foi aprovado pelo Comitê de Ética em Pesquisa do Hospital de Urgências de Goiânia (CEP/HUGO/SES) sob o protocolo no 027/11.

Inicialmente as pesquisadoras divulgaram a pesquisa nas salas de aula do Campus explicando o caráter do estudo e convidando as acadêmicas para participarem. Aquelas que se voluntariaram, tiveram um horário agendado no laboratório para realizar a coleta dos dados.

Antes das coletas, as participantes fizeram a leitura e aquelas que concordaram, assinaram o Termo de Consentimento Livre e Esclarecido. Em seguida, responderam o 
questionário epidemiológico contendo informações como nome, data de nascimento, grau de escolaridade, hábitos de vida e antecedentes patológicos, questionário Internacional de Atividade Física (IPAQ) escolhido para mensurar o nível de atividade física das participantes (FERNANI et al., 2017). O questionário de classificação socioeconômico utilizando o critério da Associação Brasileira de Empresas de Pesquisa (ABEP) para verificar a classe econômica das participantes (ABEP, 2015).

Na sequência, foi realizado exame físico constituído pela avaliação antropométrica seguindo a metodologia da International Society for the Advancement of Kinanthropometry (ISAK) (LOPES; RIBEIRO, 2014). A massa corporal (Kilogramas) e altura (centímetros), para cálculo do IMC, foram mensuradas em uma balança fixa, mecânica Welny ${ }^{\circledR}$. As participantes foram divididas em dois grupos considerando o IMC, sendo 29 mulheres eutróficas, e 25 mulheres com excesso de peso.

A avaliação antropométrica constituiu ainda das medidas das dobras cutâneas (tríceps, subescapular, supraespinhal, abdominal), em milímetros com a finalidade de calcular o percentual de gordura corporal das participantes, mediante aplicação da fórmula de predição de Falkner (HEYWARD, 2000). Para aferição das dobras cutâneas foi utilizado um adipômetro Cescorf ${ }^{\circledR}$ segundo a metodologia ISAK (LOPES; RIBEIRO, 2014).

A aferição da flexibilidade foi avaliada pelo Banco de Wells Sanny $\AA$, medindo 30,5 $\mathrm{cm} \times 30,5 \mathrm{~cm} \times 30,5 \mathrm{~cm}$ com uma escala de $26,0 \mathrm{~cm}$ em seu prolongamento. Para execução do teste, a participante retirava o calçado e na posição sentada tocava os pés na caixa com os joelhos estendidos. Com os ombros flexionados, cotovelos estendidos e mãos sobrepostas, cada participante executava a flexão do tronco à frente devendo tocar o ponto máximo da escala com as mãos. Foram realizadas três tentativas sendo considerada apenas a melhor marca (CORBETTA et al., 2008).

A força muscular de tronco foi avaliada pelo dinamômetro isocinético BIODEX $4 \mathrm{PRO}^{\circledR}$. As voluntárias foram posicionadas de acordo com as instruções definidas para este equipamento pela Biodex Medical System, Inc onde o eixo do dinamômetro foi colocado na Espinha llíaca Antero Superior e os joelhos foram posicionados com $15^{\circ}$ de flexão conforme o manual do Biodex. A avaliação da força máxima segue o seguinte protocolo: $1^{\circ}$ série é composta por uma contração isométrica de 5 segundos com o tronco fletido a $10^{\circ}$ de extensão, seguida por um descanso de 5 segundos; $2^{\circ}$ série contração isométrica de 45 segundos, com mais 5 segundos de intervalo; $3^{\circ}$ série novamente, outros 5 segundos de contração. As variáveis observadas são, pico de torque (Newton x Metro) e impulso (Newton x Metro) (CELES et al., 2013).

O desempenho funcional avaliado pelo Bunkie Test consiste em cinco posições (realizadas bilateralmente): Decúbito Dorsal Estendido (DDE), Decúbito Dorsal Fletido (DDF), Decúbito Lateral com apoio homolateral (DL), Decúbito Lateral com apoio contralateral, Decúbito Ventral (DV). Foram propostos para as participantes que assumissem a posição de teste com os membros superiores (MMSS) apoiados no chão e os membros inferiores (MMII) apoiados na mesa de tratamento, com uma altura padronizada a $30 \mathrm{~cm}$. Quando posicionadas foram dados estímulos verbais para ajudar a 
postura correta para execução do teste, na qual a posição do teste deveria ser mantida pelo máximo de tempo possível. O tempo do teste foi gravado em segundos usando um cronômetro e encerrado quando as participantes não conseguissem mais manter a posição de teste apropriada. O teste finalizou quando a participante abandonou a posição do teste apropriado (BRUMITT, 2015).

\section{ANÁLISE ESTATÍSTICA}

Os dados foram tabulados no programa Microsoft Excel® e posteriormente transferidos para o SPSS® (Statistical Package for Social Sciences) versão 23.0. Para caracterização da amostra foi utilizada estatística descritiva com medidas de tendência central e variabilidade (média e desvio padrão). Para análise de normalidade foi executado o teste de Shapiro-Wilk, sendo que todas as variáveis apresentaram distribuição normal. Desse modo, para comparar a amostra independente de dois grupos (eutróficas e excesso de peso) foi realizado o teste $t$ de Student. O nível de significância adotado foi $p<0,05$.

\section{RESULTADOS}

A amostra foi constituída por 54 mulheres com idade de 18 a 30 anos, divididas em dois grupos sendo mulheres eutróficas $(\mathrm{N}=29)$ e excesso de peso $(\mathrm{N}=25)$. As participantes eram universitárias dos cursos de Educação Física e Fisioterapia do Campus ESEFFEGO da UEG. O nível de atividade física da amostra foi avaliado pelo IPAQ e variou entre sedentárias, insuficientemente ativas, e ativas, não existindo diferença estatística entre os grupos $(p=0,945)$.

Quanto a classificação econômica pelo questionário da ABEP, as classes econômicas mais presentes foram a Classe $C$ e Classe B2, não existindo diferença estatística entre os grupos para o perfil econômico $(p=0,065)$.

A Tabela 1 evidencia os valores médios e desvio padrão para a caracterização da amostra do presente estudo.

Tabela 1 - Caracterização da amostra

\begin{tabular}{|c|c|c|c|}
\hline & \multicolumn{3}{|c|}{ Valores médios e desvio padrão } \\
\hline & Eutróficas & Excesso de peso & $p^{*}$ \\
\hline & $\mathrm{N}=29$ & $\mathrm{~N}=25$ & \\
\hline (anos) & $21,03 \pm 1,9$ & $22 \pm 3,6$ & 0,219 \\
\hline Massa corporal $\left(\mathrm{Kg}^{\star \star}\right)$ & $54,7 \pm 6$ & $78,8 \pm 10$ & $<0,001$ \\
\hline Altura $\quad\left(m^{* *}\right)$ & $1,62 \pm 0,05$ & $1,63 \pm 0,05$ & 0,60 \\
\hline IMC $\quad\left(\mathrm{kg} / \mathrm{m}^{2}\right)$ & $20,28 \pm 1,3$ & $29,38 \pm 2,8$ & $<0,001$ \\
\hline Percentual de gordura (\%) & $16,38 \pm 2,7$ & $23,97 \pm 3,2$ & $<0,001$ \\
\hline Flexibilidade - Banco de Wells $\left(\mathrm{cm}^{\star *}\right)$ & $25,29 \pm 9,9$ & $25,88 \pm 8,7$ & 0,83 \\
\hline
\end{tabular}

Nota: $\mathrm{p}^{*}$ test $t$ de Student para amostras independentes.

$\mathrm{Kg}^{* *}$ kilograma; $\mathrm{m}^{* *}$ metros; $\mathrm{cm}^{\star *}$ centímetros. Nível de significância adotado $\mathrm{p}<0,05$. 
A tabela 2 descreve os parâmetros de força muscular de tronco com valores em relação ao pico de torque e impulso.

Tabela 2 - Parâmetros de força muscular de tronco pelo dinamômetro isocinético

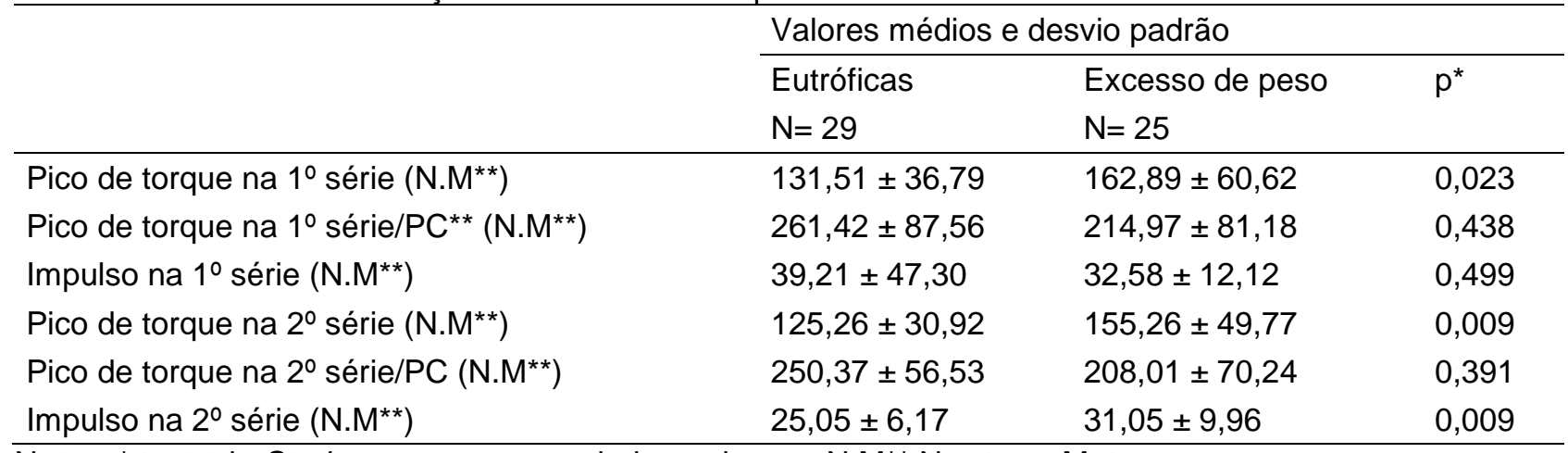

Nota: $\mathrm{p}^{*}$ test $t$ de Student para amostras independentes. N.M ${ }^{\star *}$ Newton $\times$ Metro;

$\mathrm{PC}^{* *}$ Peso Corporal. Nível de significância adotado $p<0,05$.

A funcionalidade avaliada pelo Bunkie Test está descrita na tabela 3. Observa-se que o grupo com excesso de peso teve piores resultados em todas as posições, sugerindo que o grupo de mulheres eutróficas apresentou melhor desempenho na funcionalidade.

Tabela 3 - Comparação dos parâmetros de Funcionalidade (Bunkie Test)

\begin{tabular}{|c|c|c|c|}
\hline \multirow[t]{3}{*}{ Tarefas Realizadas no Bunkie Test } & \multicolumn{3}{|c|}{ Valores médios e desvio padrão } \\
\hline & & $\begin{array}{l}\text { Excesso de } \\
\text { peso }\end{array}$ & $p^{*}$ \\
\hline & $\mathrm{N}=29$ & $N=25$ & \\
\hline Decúbito Dorsal / MID** extensão $\left(\right.$ seg $\left.^{* *}\right)$ & $15,85 \pm 10,19$ & $8,47 \pm 7,80$ & 0,011 \\
\hline Decúbito Dorsal / MIE** extensão (seg*) & $18,15 \pm 15,41$ & $7,69 \pm 5,40$ & 0,004 \\
\hline Decúbito Dorsal / MID* flexão (seg*) & $12,60 \pm 8,04$ & $10,82 \pm 9,05$ & 0,504 \\
\hline Decúbito Dorsal / MIE * flexão (seg*) & $13,90 \pm 9,89$ & $9,34 \pm 9,37$ & 0,130 \\
\hline Decúbito Lateral Direito / homolateral (seg*) & $13,65 \pm 10,47$ & $6,42 \pm 5,43$ & 0,008 \\
\hline Decúbito Lateral Direito / contralateral (seg*) & $7,78 \pm 6,70$ & $6,04 \pm 5,55$ & 0,368 \\
\hline Decúbito Lateral Esquerdo / homolateral (seg*) & $10,50 \pm 8,40$ & $6,71 \pm 5,30$ & 0,091 \\
\hline Decúbito Lateral Esquerdo / contralateral (seg*) & $8,31 \pm 8,41$ & $6,13 \pm 5,35$ & 0,322 \\
\hline Decúbito Ventral / MID* extensão $\left(\right.$ seg $\left.^{*}\right)$ & $10,20 \pm 6,21$ & $8,50 \pm 6,06$ & 0,375 \\
\hline Decúbito Ventral / MIE* extensão (seg*) & $10,85 \pm 5,76$ & $8,0 \pm 5,01$ & 0,094 \\
\hline
\end{tabular}

Nota: $\mathrm{p}^{*}$ test $t$ de Student para amostras independentes; ${ }^{* *}$ MID Membro inferior direito, MIE Membro inferior esquerdo; **seg Segundos. Nível de significância adotado $p<0,05$

\section{DISCUSSÃO}

O presente estudo objetivou verificar a influência do excesso de peso corporal na força muscular de tronco e funcionalidade de mulheres jovens. A amostra do presente estudo foi composta por mulheres jovens universitárias com idade 19 a 30 anos. Dados similares para a idade são encontrados em estudos que caracterizam a população de universitárias conforme evidenciado por Silva et al. (2015) e Souza et al. (2012), que encontraram respectivamente, universitárias com idade média de 20,42 anos, e idade 
média foi de 20,9 anos.

O nível de atividade física da amostra foi avaliado pelo IPAQ, teve como resultado que variou entre sedentárias e ativas, não existindo diferença estatística entre os grupos. Este achado concorda com estudo de Souza et al. (2015) que avaliou acadêmicos dos cursos da área da saúde e encontrou a frequência percentual similar de fisicamente inativos e de ativos entre todos os cursos da área da saúde investigados. Achado semelhante foi encontrado no estudo de Gasparotto et al. (2013), que encontrou no seu estudo $54,5 \%$ dos universitários não seguiam as recomendações de atividade física enquanto que 45,5\% classificados como ativos.

Quanto ao perfil socioeconômico da amostra identificou-se que as classes econômicas mais presentes foram a Classe C e Classe B2, não existindo diferença estatística entre os grupos. Sendo semelhante ao perfil socioeconômico do estudo de Ferreira e Benicio (2015) no que tange às classes de poder aquisitivo de mulheres em idade reprodutiva entre 15 a 49 anos de idade, 46,3\% das participantes pertenciam à classe média (classe C), colaborando também com a investigação Gasparotto et al. (2013), que avaliou 1.631 alunos, distribuídos em calouros e veteranos, mostra a prevalência da classe econômica B.

A amostra do presente estudo foi caracterizada como excesso de peso a partir do cálculo do IMC. A variável altura não apresentou diferença estatística entre os grupos, demostrando que o excesso de peso da amostra estudada sofreu variação somente pela alteração do peso corporal. Dessa maneira a variável IMC, que é composta pela massa corporal dividida pelo quadrado a altura, não sofreu influência da altura, resultado semelhantemente aos estudos Silva-Hamu et al. (2013) e Castro et al. (2018).

Ainda quanto a caracterização do excesso de peso, o percentual de gordura da presente amostra apresentou diferença estatística entre os grupos, apresentando relação direta com o IMC. As medidas antropométricas obtidas pela técnica de dobras cutâneas (DC) são precisas para detecção do percentual de gordura (\% GC), que são parâmetros bastante utilizados para identificar o excesso de peso (SALES et al. 2013). No estudo de Grossl et al. (2010) observou-se que o IMC foi o indicador que teve maior correlação com ० \% GC. Expressando uma relação positiva entre ० \% GC e os indicadores antropométricos analisados.

Ao se avaliar a flexibilidade e identificar se a mesma sofre influência do excesso de peso, os achados do presente estudo não encontraram diferenças para a flexibilidade entre os dois grupos. Achados semelhantes também foram encontrados na pesquisa que tiveram como objetivo relacionar o IMC com os níveis de flexibilidade, tendo a hipótese de que o sobrepeso pudesse influenciar em baixos níveis de flexibilidade. No estudo com amostra composta por 82 alunos de 13 a 17 anos de idade, a avaliação da flexibilidade por meio do teste sentar-e-alcançar indicou que os níveis de flexibilidade não foram influenciados pelo índice de massa corporal (CEZAR e CEZAR, 2014). Concordando ainda com Ferreira et al. (2013), que utilizou o mesmo protocolo com amostra de estudantes de ambos os sexos, idade entre 14 a 18 anos, e identificou que o peso corporal não interferiu nos resultados do 
teste da flexibilidade.

Quanto a avaliação da flexibilidade, o presente estudo constatou que o excesso de peso não influenciou a mesma. Sabe-se que a flexibilidade dos músculos isquiotibiais é fundamental para manutenção do equilíbrio postural e amplitude de movimento adequada do tronco, quadril e joelho. Os músculos isquiotibiais desempenham uma forte relação com os músculos paravertebrais, podendo gerar disfunções na coluna lombar. Quando encurtados apresentam uma diminuição da flexibilidade, aumentam a lordose lombar além de gerar uma contração dos músculos paravertebrais. Se em comprimento normal o movimento torna-se fisiológico, melhorando o desempenho muscular e prevenindo os distúrbios e desequilíbrios musculares (DORTA, 2016). No presente estudo, foi fundamental que não houvesse diferença na flexibilidade dos músculos isquiotibiais entre os grupos. Deste modo, o movimento de tronco não sofreria influência de uma possível diferença de flexibilidade.

Quanto aos resultados referentes à avaliação da força muscular de tronco, o estudo de Cavuoto e Nussbaum (2013), com 36 participantes (18 a 29 anos) objetivou ampliar as evidências disponíveis em relação à força e desempenho funcional. A amostra foi composta por dois grupos de não obesos e obesos. A avaliação da força muscular foi realizada pelo dinamômetro (Biodex System 3 Pro), no qual os participantes ficaram na posição vertical, e foram instruídos a realizar uma força máxima, mantê-la e, em seguida, descansar por 5 s. Os resultados mostram que o tempo de resistência e as demandas da tarefa (como uma porcentagem da força) foram semelhantes nos dois grupos. Concluiu que a obesidade pode não influenciar substancialmente a capacidade muscular de tronco.

O mesmo estudo relatou que os participantes com excesso de peso apresentaram uma diferença na percepção da fadiga, mostrado que os músculos ficam cansados e consequentemente apresentam uma falha na manutenção de contração isométrica. Concluindo que durante isométricos os participantes apresentaram uma influência significativa para fadiga. Concordando com o presente estudo que encontrou essa mesma variável da fadiga, onde observou que existe uma maior relação da fadiga nos indivíduos com excesso de peso (CAVUOTO; NUSSBAUM, 2013).

O estudo Pajoutana et al. (2016), estudou 68 universitários que foram divididos pelo IMC, realizaram um protocolo no dinamômetro isocinético (Cybex), no qual os indivíduos ficavam de pé e levemente inclinado para frente, realizando uma força máxima isométrica de extensão durante 5 segundos, seguida de um descanso de 2 minutos entre os esforços, sendo repetido três vezes. Revelou uma diferença significativa entre os grupos, concluindo que a força de tronco foi menor no grupo com excesso de peso. O que implica o comprometimento da obesidade na capacidade funcional do músculo tronco. Porém esses resultados não estão de acordo com os do presente estudo, quando analisados os picos de torque sem a relação com o peso corporal.

No presente estudo, ao se analisar o torque normalizado pelo peso corporal, encontrou-se que mesmo não havendo diferença significativa entre os grupos, as mulheres com excesso de peso produzem um torque menor que as mulheres eutróficas. No estudo 
de Pajoutana et al. (2016), quando a força foi normalizada pele peso corporal foram encontradas diferenças significativas entre os grupos de excesso de peso e eutroficos, demostrando que força foi menor no grupo com excesso de peso. Cavuoto e Nussbaum (2013), ao analisar a normalização dos dados não encontrou diferença estatística entre os grupos, mas revelou que os participantes com peso normal apresentaram um pico de torque maior.

De acordo com achados de Hulens et al. (2002), que avaliaram 241 mulheres obesas (18-65 anos), na qual o objetivo foi identificar variáveis que afetassem a força muscular avaliados pelo pico de torque no dinamômetro Cybex 350. Identificou-se que o pico de torque de todas as medidas de resistência apresentou uma associação positiva com massa corporal, altura, massa livre de gordura, explicando a maior parte da variância de força.

No estudo de Tomlinson et al. (2014), com uma população 100 mulheres (17-48 anos), foi relatado nos indivíduos com excesso de peso um aumento da força isométrica, que pode ser explicado pelo aumento do IMC e da adiposidade, associado ainda ao aumento do volume muscular esquelético. Sendo que as mulheres jovens tem a massa muscular capaz de se adaptar ao próprio carregamento de altos níveis de IMC e / ou adiposidade consequentemente aumentando o volume muscular gerando então por si só torque maior. Concluindo que indivíduos com excesso de peso, têm uma força muscular máxima pelo aumento da adiposidade que atua como um estímulo de sobrecarga crônica nos músculos antigravitacionais, aumentando a força.

Foi verificado também com estudo de Lafortuna et al. (2014), que o aumento da adiposidade do corporal sofreu uma influência positiva sobre essas características musculares. Portanto, podendo explicar os possíveis resultados encontrados no presente estudo.

O Bunkie test é descrito no estudo de Witt e Venter (2009), como um teste preciso para medir a funcionalidade dos músculos do tronco, método bastante utilizado nas avaliações de atletas para aferir a função das linhas de fáscia. Os resultados mostraram ao longo dos anos que este teste pode ser usado com sucesso para encontrar a causa e tratar lesões e determinar o progresso do tratamento em atletas de elite e recreação. A fáscia muscular e o músculo quando perdem sua mobilidade normal podem gerar restrição de movimento, e se encurtados ou em alongamento excessivo, resulta em menos estabilidade e fraqueza não somente muscular, mas também alterações estruturas articulares, ligamentares e óssea.

O presente estudo torna-se o primeiro a avaliar a capacidade funcional pelo Bunkie test em mulheres jovens com excesso peso e comparando-as com mulheres eutróficas. Visto que os dados mais encontrados na literatura são com população saudável (BRUMITT, 2015). Quando comparadas as mulheres com excesso de peso e as eutróficas, foram encontrados resultados menores em todas as posições, portanto identificando uma diminuição no desempenho da funcionalidade das participantes com excesso de peso. 
Com relação à funcionalidade o estudo de Brumitt (2015) avaliou indivíduos saudáveis pelo Bunkie test, sendo 81 mulheres e 31 homens. $O$ estudo verificou que as mulheres com IMC menor foram capazes de manter a posição mais tempo do que aquelas com maior IMC, para as posições de DD com membro inferior em flexão no lado direita. Já os homens com IMC menor puderam manter 8 das 10 posições por mais tempo do que os homens com IMC maior. Na posição de DL direito homolateral, em homens, revelou-se um tempo de espera assimétrico que correlacionou a fraqueza do lado esquerdo do glúteo máximo, glúteo médio e rotadores externos do quadril, explicando os resultados do teste.

Dentre as posições que apresentaram diferença estatística estão o teste de DD com MMII estendido que se refere à estabilização posterior (músculos lombares incluindo a fáscia toracolombar, glúteo máximo, posterior de coxa e gastrocnêmio), e teste de DL direito homolateral referente à estabilização lateral (oblíquos, glúteo médio, tensor da fáscia lata, gastrocnêmio e soleo), podendo indicar desequilíbrio muscular, podendo ser justificado pela própria fraqueza muscular, consequentemente diminuindo o desempenho funcional dessas participantes. Concordando com Brumitt (2011), que encontrou no seu estudo tempos de espera assimétricos correlacionados com fraqueza do lado esquerdo do glúteo máximo, glúteo médio e rotadores externo, para o teste de DD direito homolateral.

\section{CONSIDERAÇÕES FINAIS}

O presente estudo identificou que as mulheres com excesso de peso conseguem gerar maior pico de torque absoluto que as mulheres eutróficas. Mas, quando o pico de torque é analisado proporcionalmente ao peso corporal, o pico de torque é menor, indicando uma possível influência do IMC na força muscular de tronco. Demonstrou-se que as mulheres com excesso de peso apresentaram pior desempenho na funcionalidade pelo Bunkie Test, sugerido um desequilíbrio muscular em tronco e membros inferiores. Portanto, considera-se que o excesso de peso pode alterar diretamente a força muscular e interferir na funcionalidade de tronco de mulheres jovens.

\section{REFERÊNCIAS}

Associação Brasileira de Empresas de Pesquisas. Critério Brasil 2015 e atualização da distribuição de classes para 2016. São Paulo, 2015. Disponível em: <http://www.abep.org/criterio-brasil>. Acesso em: 17 outubro 2017.

BATTISTI, Larissa. et al. Percepção da qualidade de vida e funcionalidade em obesos candidatos a cirurgia bariátrica: um estudo transversal. Revista Brasileira de Qualidade de Vida, Ponta Grossa, v. 9, n. 2, p. 125-140, jan. 2017.

BRUMITT, Jason. Successful rehabilitation of a recreational endurance runner: initial validation for the Bunkie test. Journal of Bodyworkand Movement Therapies, Hillsboro, v. 15, n. 3, p. 384-390, maio. 2011. 
BRUMITT, Jason. The Bunkie Test: Descriptive Data for a Novel Test of Core Muscular Endurance. Rehabilitation Research and Practice, Newberg, v. 123, n. 414, p. 9, jan. 2015.

CASTRO, Jéssica Marliere de. et al. Prevalência de sobrepeso e obesidade e os fatores de risco associados em adolescentes. Revista Brasileira de Obesidade, Nutrição e Emagrecimento, São Paulo, v. 12, n. 69, p. 84-93, jan./fev. 2018.

CAVUOTO, Lora A; NUSSBAUM, Maury A. Obesity-related differences in muscular capacity during sustained isometric exertions. Applied Ergonomics, Blacksburg, v. 44, p. 254-260, mar. 2013.

CELES, Rodrigues. et al. Fadiga muscular entre séries de exercícios isocinéticos em mulheres jovens. Motriz, Rio Claro, v. 19, n. 2, p. 494-501, abr./jun., 2013.

CEZAR, Juliê Rebellatto; CEZAR Marcos Antônio. Relação entre níveis de flexibilidade e composição corporal de jovens praticantes de futebol. Unoesc \& Ciência, p. 121-128, 2014.

CORBETTA, Angélica Rui. et al. Os testes de flexibilidade do banco de wells realizados em jovens no processo de recrutamento obrigatório demonstraram que a atividade física não influencia na flexibilidade muscular. Revista Brasileira de Prescrição e Fisiologia do Exercício, São Paulo, v. 2, n. 10, p. 409-414, jul./ago. 2008.

DORTA, Haron Silva. Relação entre os músculos isquiotibiais e paravertebrais Com a lombalgia. Coluna/Columna, São Paulo, v. 15, n. 3, p. 241-243, mar. 2016.

FERNANI, Deborah Cristina Gonçalves Luiz. et al. Anthropometric profile, physical activity level, degree of anxiety, and posture in college students. Revista Fisioterapia e Pesquisa, v. 24, n. 2, p. 191-197, jun. 2017.

FERREIRA, Marcus Vinicius Nascimento. et al. A prevalência da obesidade e sobrepeso e níveis de flexibilidade em adolescentes de 14 a 18 anos. Fédération Internationale d'Education Physique, v. 83, 2013.

FERREIRA, Regicely Aline Brandão; BENICIO, Maria Helena D'Aquino. Obesidade em mulheres brasileiras: associação com paridade e nível socioeconômico. Revista Panamericana de Salud Pública, São Paulo, v. 37, n. 4/5, 2015.

GASPAROTTO, Guilherme da Silva. et al. Associação entre fatores sociodemográficos e o nível de atividade física de universitários. Revista Brasileira de Ciência e Movimento, v. 21 , n. 30 , p. $30-40$, out. 2013.

GROSSL, Talita; LIMA, Luiz Rodrigo Augustemak de; KARASAIAK, Fábio Colussi. Relação entre a gordura corporal e indicadores antropométricos em adultos frequentadores de academia. Motricidade, Florianópolis, v. 6, n. 2, p. 35-45, mar. 2010.

HEYWARD, Vivian H; STOLARCZYK, Lisa M. Avaliação da composição corporal aplicada. São Paulo: Manole. 2000.

HULENS, Maria. et al. Assessment of Isokinetic Muscle Strength in Women Who Are Obese. Journal of Orthopaedic \& Sports Physical Therapy, Belgium, v. 32, n. 7, p. 
347-356, jul. 2002.

LAFORTUNA, Claudio L; TRESOLDI, Daniele; RIZZO, Giovanna. Influence of body adiposity on structural characteristics of skeletal muscle in men and women. Clinical Physiology and Functional Imaging, Milan, v. 34, p. 47-55, jan. 2014.

LOPES, André Luiz; RIBEIRO, Gustavo dos Santos. Antropometria aplicada à saúde e ao desempenho esportivo: uma abordagem a partir da metodologia ISAK. $1^{\circ}$ ed. Rio de Janeiro: Rubio, 2014, 232 p.

LOPES, Geovanna Lemos; SANTOS, Maria Izabel Penha de Oliveira. Funcionalidade de idosos cadastrados em uma unidade da Estratégia Saúde da Família segundo categorias da Classificação Internacional de Funcionalidade. Revista Brasileira de Geriatria e Gerontologia, Rio de Janeiro, v. 18, n. 1, p. 71- 83, jan./mar. 2015.

LOPES, Wendell Arthur. et al. Influência da obesidade na força muscular de membros inferiores e superiores em adolescentes. Revista Brasileira de Atividade Física \& Saúde, Pelotas, v.18, n.6, nov. 2013.

NEW YORK. Dual position back ex/flex attachment: Operation Manual. Biodex Medical Systems, Inc. Disponível em:

<http://www.biodex.com/sites/default/files/830450man_08261reva.pdf>. Acesso em 31 de outubro de 2017.

PAJOUTANA, Mojdeh; MEHTA, Ranjana K; CAVUOTO, Lora A. Obesity effect on isometric strength of the trunk extensors. Journals Sage, Texas, 2016.

PHILLIPS, Catherine M. Metabolically healthy obesity across the life course: epidemiology, determinants, and implications. Annals of the new york academy of sciences, New York, v. 1391, mar. 2016.

SANT'ANNA JUNIOR, Maurício de. et al. Disfunção Autonômica Cardiovascular em Pacientes com Obesidade Mórbida. Arquivos Brasileiros de cardiologia, Rio de Janeiro, v. 105, n. 6, p. 580-587, nov. 2015.

SALES, Marcelo Magalhães. et al. Índice de massa corporal estima percentual de gordura corporal calculado pela espessura de dobras cutâneas em mulheres adultas. Revista Brasileira de Ciência e Movimento, v. 21, n. 2, p. 5-10, nov. 2013.

SILVA-HAMU, Tânia Cristina Dias da. et al. The impact of obesity in the kinematic parameters of gait in young women. International Journal of General Medicine, Goiânia, v. 6, p. 507-513, jun. 2013.

SILVA NETO, Moacir. Avaliação isocinética da força muscular em atletas profissionais de futebol feminino. Revista Brasileira de Medicina do Esporte, Niterói, v. 16, n. 1, jan./feb. 2010.

SILVA, Wanderson Roberto. et al. Fatores que contribuem para preocupação com a imagem corporal de estudantes universitárias. Revista Brasileira de epidemiologia, Araraquara, v. 18, n. 4, p. 785-797, out./dez. 2015.

SIQUEIRA, Gisela Rocha; SILVA, Giselia Alves Pontes da. Alterações posturais da coluna 
e instabilidade lombar no indivíduo obeso: uma revisão de literatura. Revista Fisioterapia em Movimento, v. 24, n. 3, p 557-66, jul/.set. 2011.

SOUZA, Ivo Eduardo Chanca Diniz de. et al. Níveis de atividade física e estágios de mudança de comportamento de universitários da área de saúde. Revista Brasileira de Atividade Física \& Saúde, Pelotas, v. 20, n. 6, p. 608-610, nov. 2015.

SOUZA, Luciana Bronzi de. et al. Inadequação de consumo alimentar, antropometria e estilo de vida de universitárias da área de saúde. Journal of the Health Sciences Institute, Botucatu, v. 30, n. 4, p. 377-381, 2012.

TOMLINSON, Dave J. et al. The impact of obesity on skeletal muscle architecture in untrained young vs. old women. Journal of Anatomy, v. 225, p. 675-684, oct. 2014.

TOSCANO, José Jean de Oliveira; EGYPTO, Evandro Pinheiro do. A influência do sedentarismo na prevalência de lombalgia. Revista Brasileia de Medicina do Esporte, Niterói v. 7, n. 4, jul./aug. 2001.

ZABKA, Felipe Furlan; VALENTE, Henrique Gonçalves; PACHECO, Adriana Moré. Avaliação isocinética dos músculos extensores e flexores de joelho em jogadores de futebol profissional. Revista Brasileira de Medicina do Esporte, São Paulo, v.17, n. 3 maio./jun. 2011.

WITT, Benita de; VENTER, Ranel. The 'Bunkie' test: Assessing functional strength to restore function through fascia manipulation. Journal of Bodywork and Movement Therapies, v. 13, p. 81-88, jan. 2009.

World health organization. Obesity and overweight. Genebra, 2017. Disponível em: <http://www.who.int/mediacentre/factsheets/fs311/en/>. Acesso em: 25 de outubro 2017. 